\title{
Elevated levels of soluble fractalkine and increased expression of CX3CR1 in neuropsychiatric systemic lupus erythematosus
}

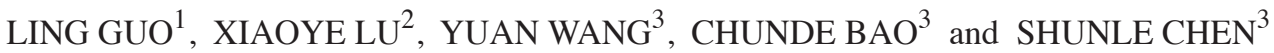 \\ ${ }^{1}$ Department of Rheumatology, Dongying People's Hospital, Dongying, Shandong 257091; \\ Departments of ${ }^{2}$ Emergency and ${ }^{3}$ Rheumatology, Renji Hospital, School of Medicine, \\ Shanghai Jiaotong University, Shanghai 200001, P.R. China
}

Received June 26, 2015; Accepted August 23, 2016

DOI: $10.3892 /$ etm.2017.4862

\begin{abstract}
The aim of the present study was to determine the levels of soluble fractalkine (sFKN) and expression of CX3CR1 in neuropsychiatric systemic lupus erythematosus (NPSLE). Disease activity of SLE was assessed using the SLE Disease Activity Index (SLEDAI). The mRNA expression levels of CX3CR1 and FKN were quantified using reverse transcription-quantitative polymerase chain reaction. Levels of sFKN in the serum and cerebrospinal fluid (CSF) were measured using enzyme-linked immunosorbent assays. The mRNA expression levels of CX3CR1 in peripheral blood mononuclear cells from patients with NPSLE, non-NPSLE and Behcet's disease were significantly higher than that of rheumatoid arthritis and healthy persons. Levels of sFKN in the serum and CSF of cells with diffuse NPSLE (DNPSLE) were significantly higher than those of focal NPSLE (FNPSLE) cells. Serum levels of sFKN were higher in patients with NPSLE or non-NPSLE than heathy persons. sFKN in CSF were significantly higher in DNPSLE than non-NPSLE cells, but there were no significant difference between FNPSLE and control. Treatment reduced sFKN in serum and CSF in patients with NPSLE. There was significant correlation between $\mathrm{sFKN}$ in the serum of patients with SLE and the SLEDAI. sFKN levels were correlated with IgG in CSF from patients with NPSLE. The mRNA expression levels of CX3CR1 in the brain tissue of lupus mice were significantly higher than normal mice; however, the mRNA expression of FKN was lower than normal mice. These results suggest that sFKN and CX3CR1 may be involved in vasculitis and SLE, particularly in DNPSLE, which may occur by damaging the blood-brain barrier or recruiting expression microglial cells of CX3CR1. Additionally, sFKN appears to be
\end{abstract}

Correspondence to: Dr Ling Guo, Department of Rheumatology, Dongying People's Hospital, 317 First South Road, Dongying, Shandong 257091, P.R. China

E-mail: guoling0920@126.com

Key words: soluble fractalkine, CX3CR1, neuropsychiatric systemic lupus erythematosus, mRNA expression a serological marker in patients with SLE, and may be useful for the diagnosis and treatment of NPSLE.

\section{Introduction}

Systemic lupus erythematosus (SLE) is a systemic autoimmune disease in which the body's immune system mistakenly attacks healthy tissue (1). When SLE affects the central or peripheral nervous systems, it is called neuropsychiatric systemic lupus erythematosus (NPSLE) (2). The American College of Rheumatology (ACR) defines 19 neuropsychiatric syndromes in SLE in 1999, and NPSLE was divided into three clinical types: Diffuse (DNPSLE), focal (FNPSLE) and peripheral neurological SLE (2). Manifestations of DNPSLE include cognitive dysfunction, acute confusion states, psychosis, mood and anxiety disorders (2). Manifestations of FNPSLE include seizures, cerebrovascular disease, headache, demyelinating syndrome, aseptic meningitis, chorea and myelopathy (2). Autoantibody and inflammatory cells are important pathogenic factors of NPSLE. Infiltration and activation of inflammatory cell often require the involvement of chemotactic factors (3). Fractalkine $(\mathrm{FKN})$ is the only known member of the $\mathrm{CX} 3 \mathrm{C}$ family at present which is a recently discovered chemotactic factor, the chemokine receptor of which is CX3CR1 (4). FKN is primarily expressed and secreted by activated vascular endothelial cells, epithelial cells, synovial cells, and is highly expressed in "membrane-anchored" and "free" neurons. Studies of FKN regarding SLE are more concentrated in lupus nephritis (LN), while the role of FKN in NPSLE is less understood $(5,6)$. Thus, the aim of the present study was to assess the mRNA expression of CX3CR1 in peripheral blood mononuclear cells (PBMCs), and levels of sFKN in the serum and cerebrospinal fluid (CSF) to investigate the involement of sFKN in NPSLE. In addition, the mRNA expression levels of FKN and CX3CR1 in the brain tissue of lupus mice were evaluated to determine their effect on NPSLE.

\section{Materials and methods}

Patients. Subjects admitted to Renji Hospital (Shanghai, China) were retrospectively analyzed from November 2005 to October 2008, including 152 patients and 51 healthy persons (HPs). The patient population consisted of non-NPSLEs 
(female, 29; male, 5; age, 29 7 years), RA (female, 26; male, 4; age, $30 \pm 5$ years), BD (female, 38; male, 10; age, $32 \pm 7$ years). Healthy control population consisted of 43 females and 8 males (age, $31 \pm 6$ years). In addition, brain tissues from 8 lupus mice were analyzed, obtained from the Model Animal Research Center, Nanjing University (Nanjing, China). Patients with SLE conformed to the standard of classification revised by ACR in 1997 (1), while NPSLE conformed to the class definition proposed by the ACR in 1999 (2). Neuropsychiatric symptoms include: i) Abnormal electroencephalogram, which are predominantly with slow wave and diffuse activity; ii) abnormal CSF, which mainly manifested as increased pressure, total protein, white blood cell count and/or total IgG; and iii) abnormal computed tomography or magnetic resonance imaging. Infection, drugs and metabolic factors were excluded; such as infection/drugs/metabolic factors including intracranial tuberculosis, bacteria and streptococcus, the nerve symptoms caused by drugs such as corticosteroids, low sodium and low chlorine encephalopathy. Furthermore, rheumatoid arthritis (RA) was identified according to the diagnostic criteria of ACR in 1987 (7) and Behcet's disease (BD) according to the diagnostic criteria of the International Study Group for Behcet's disease in 1990 (8). This study was approved by the ethics committee at Dongying People's Hospital and was conducted in accordance with the provisions of the Declaration of Helsinki, Good Clinical Practice guidelines, and local laws and regulations.

mRNA expression levels of CX3CR1 in PBMCs. Investigated subjects include 40 cases with NPSLE, 34 cases with SLE but no neuropsychiatric symptoms (non-NPSLE), 30 cases with RA, 48 cases with BD and 51 HPs. Average age of the NPSLE group was $33 \pm 14$ years old, including 16 cases with DNPSLE and 24 cases with FNPSLE. Leucocytes from the peripheral blood of above investigated subjects were collected and dissolved in TRIzol to extract total RNA. Integrity, purity and concentration were tested (9), then total RNA was reverse transcribed to cDNA. mRNA expression of CX3CR1 was quantified using reverse transcription-quantitative polymerase chain reaction, using a SYBR Premix Ex Taq kit (Takara Bio, Inc, Otsu, Japan). Primers were designed as following: Reference gene RPL13A forward, 5'-CCTGGAGGAGAA GAGGAAAGAGA-3' and reverse 5'-TTGAGGACCTCT GTGTATTTGTCAA-3'; CX3CR1 forward, 5'-AGCAGGCAT GGAAGTGTTCT-3' and reverse 5'-GTTGTTTTGTGTGCA TTGGG-3'. Primers were stored at $-20^{\circ} \mathrm{C}$ after dissolution and dilution. The operation was performed according to the instructions of a DRR041A Takara Kit (Takara Bio, Inc.). A 5- $\mu 1$ reaction system included 2.5 $\mu \mathrm{l}$ 2X SYBR Premix, $0.1 \mu \mathrm{l}$ Forward Primer $(10 \mu \mathrm{M}), 0.1 \mu \mathrm{l}$ Reverse Primer $(10 \mu \mathrm{M}), 0.1 \mu \mathrm{l}$ ROX Reference Dye, $1.2 \mu \mathrm{l}$ deionized water and $1.0 \mu \mathrm{l} \mathrm{cDNA}$. Reaction plates were centrifuged at 2,000 rpm for 1 min at $4^{\circ} \mathrm{C}$. PCR was performed according to the instruction of ABI 7900 Sequence Detection System (Applied Biosystems; Thermo Fisher Scientific, Inc., Foster City, CA, USA). Reaction conditions are set as follows: Pre denaturation $\left(95^{\circ} \mathrm{C}\right.$ for $10 \mathrm{sec}$, Reps: 1), PCR $\left(95^{\circ} \mathrm{C}\right.$ for $5 \mathrm{sec}, 60^{\circ} \mathrm{C}$ for $30 \mathrm{sec}$, Reps: 40$)$ and a dissociation Stage with the same parameter. Sample volume was $5 \mu 1.2^{-\Delta \Delta \mathrm{Ct}}$ value was considered to be proportional to the mRNA expression of the corresponding gene in the specimen.
Levels of sFKN in the serum and CSF. Serum samples were collected from 30 NPSLE patients, 28 female and 2 male, including 13 cases with DNPSLE and 17 cases with FNPSLE (average age, $34 \pm 13$ years). Serum samples were collected from 53 non-NPSLE cases, 49 female and 4 male (average age, $33 \pm 11$ years). Serum samples were additionally collected from $39 \mathrm{HPs}$, including 37 females and 2 males (average age, $34 \pm 8$ years). CSF samples were collected only from 8 cases with DNPSLE and 15 cases with active stage FNPSLE. In addition, serum samples from 17 cases and CSF of 8 cases with NPSLE were collected after treatment with 200-1,000 mg methylprednisolone per day for 3-5 days (Pfizer Manufacturing Belgium NV, Puurs, Belgium) and immunosuppressive drugs including cyclophosphamide 0.4-0.6 g per week and hydroxychloroquine sulfate $0.1-0.2 \mathrm{~g}$ bid (Baxter Oncology GmbH, Halle, Germany), for 1-2 weeks. Dosage and course of treatment were determined according to the severity of the illness and the patient's weight. For ethical reasons, CSF samples were not collected from HPs. As the inspection results of SLE patients did not conform to the diagnostic criteria of NPSLE, CSF from these SLE patients was considered to be non-NPSLE. Age and gender of all above groups had no statistically significant difference. The active disease stage of each patient was determined according to the SLE Disease Activity Index (SLEDAI), with an SLEDAI >10 indicating the active stage (10). The concentration of total IgG in the CSF of cases with NPSLE was obtained from patient medical records. Levels of sFKN in serum and CSF were evaluated using an enzyme-linked immunosorbent assay (DY365) and substrate kit (DY999; R\&D Systems, Inc., Minneapolis, MN, USA).

mRNA expression of FKN and CX3CR1 in the brain tissue of lupus mice. Investigated samples consisted of brain tissues from 8 lupus mice, including 3 NZB, 1 NZW, 2 NZB/NZWF1 and 2 BXSB mice ( 6 females and 2 males). Six matched normal mice were also included (B57; Shanghai Institute for Biological Sciences, Chinese Academy of Sciences, Shanghai, China). The rats were anesthetized with 0.4-0.6 ml $0.25 \%$ pentobarbital Sodium (Sigma-Aldrich; Merck KGaA, Darmstadt, Germany) and sacrificed by cutting the abdominal cavity and thoracic cavity, exposing the heart and then blood letting. Brain tissues of mouse were obtained through operation and stored in a refrigerator. Brain tissues were ground in liquid nitrogen to dissociate cells. After removing impurity, total RNA was extracted to determine the mRNA expression levels of FKN and CX3CR1. Primers were designed as follows: $\beta$-actin, forward 5'-ATGCTCCCCGGGCTGTAT-3' and reverse 5'-CATAGGAGTCCTTCTGACCCATTC-3'; mouse CX3CR1, forward 5'-TGTTATTTGGGCGACATTG-3' and reverse 5'-CGAGGACCACCAACAGATT-3'; and mouse FKN, forward 5'-TGCGACAAGATGACCTCAC-3' and reverse 5'-TCTGTGCTGTGTCGTCTCC-3'. PCR protocols were the same as thosed use for detecting CX3CR1.

Statistical analysis. SPSS software, version 19.0 (IBM SPSS, Armonk, NY, USA) was used to analyze data. Data were manipulated using normal distribution and homogeneity of variance tests, then with one-way analysis of variance or non-parameter statistics. All data are expressed as the mean \pm standard error. Data from before or after treatment 


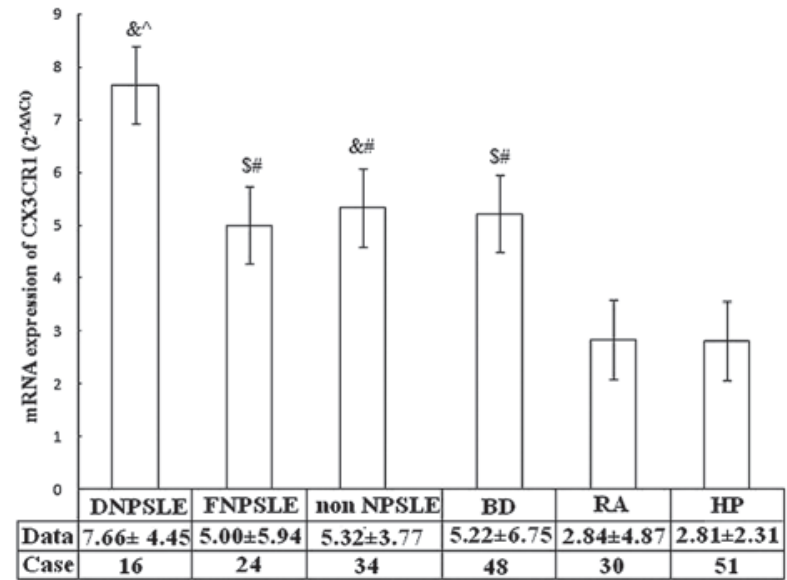

Figure 1. mRNA expression of CX3CR1 in PBL. \& $\mathrm{P}<0.01$ vs. HP; ${ }^{\$} \mathrm{P}<0.05$ vs. HP; ${ }^{\wedge} \mathrm{P}<0.01$ vs. RA; ${ }^{\#} \mathrm{P}<0.05$ vs. RA. DNPSLE, diffuse neuropsychiatric systemic lupus erythematosus; FNPSLE, focal neuropsychiatric systemic lupus erythematosus; BD, Behcet's disease; RA, rheumatoid arthritis; HP, healthy person.

were compared using $t$-test. Correlation was performed using Pearson correlation analysis. $\mathrm{P}<0.05$ were considered to indicate a statistically significant difference.

\section{Results}

mRNA expression levels of CX3CR1 in PBMCs. The mRNA expression levels of CX3CR1 in the PBMCs of NPSLE, non-NPSLE and BD patients were all significantly higher than the RA and HP PBMCs. However, no statistically significant differences were detected among the DNPSLE, FNPSLE, non-NPSLE and BD groups (Fig. 1).

Levels of $S F K N$ in the serum and CSF. Levels of sFKN in serum samples from patients with DNPSLE were significantly higher than those from FNPSLE patients. In addition, the DNPSLE and FNPSLE groups had significantly higher sFKN levels than non-NPSLE and HP groups, whereas non-NPSLE was only significantly higher than HP (Fig. 2). sFKN in CSF were significantly higher in DNPSLE compared with FNPSLE and non-NPSLE $(\mathrm{P}<0.01)$, but there were no significant differences between FNPSLE and non-NPSLE (Fig. 3). Moreover, IgG in CSF samples from DNPSLE $(31.3 \pm 28.8 \mathrm{mg} / \mathrm{dl})$ were higher than FNPSLE $(7.1 \pm 6.6 \mathrm{mg} / \mathrm{dl})$ (data not shown).

Correlation between sFKN and patient treatment, SLEDAI and $\operatorname{IgG}$. Comparisons of levels of sFKN in the serum and CSF before and after treatment with methylprednisolone and immunosuppressive drugs are shown in Figs. 4 and 5. Before treatment, sFKN in serum and CSF were 5,232 $\pm 3,039$ and $2,632 \pm 2,272 \mathrm{pg} / \mathrm{ml}$, respectively. However, after treatment the data became $2,414 \pm 2,507$ and $705 \pm 404 \mathrm{pg} / \mathrm{ml}$, respectively. sFKN in the serum of patients with SLE were positively correlated with SLEDAI ( $r=0.671, \mathrm{P}<0.01$; Fig. 6). In addition, sFKN in CSF from patients with NPSLE were positively correlated with $\operatorname{IgG}(r=0.945, \mathrm{P}<0.01$; Fig. 7$)$.

mRNA expression of FKN and CX3CR1 in the brain tissue of lupus mice. The mRNA expression levels of CX3CR1 in

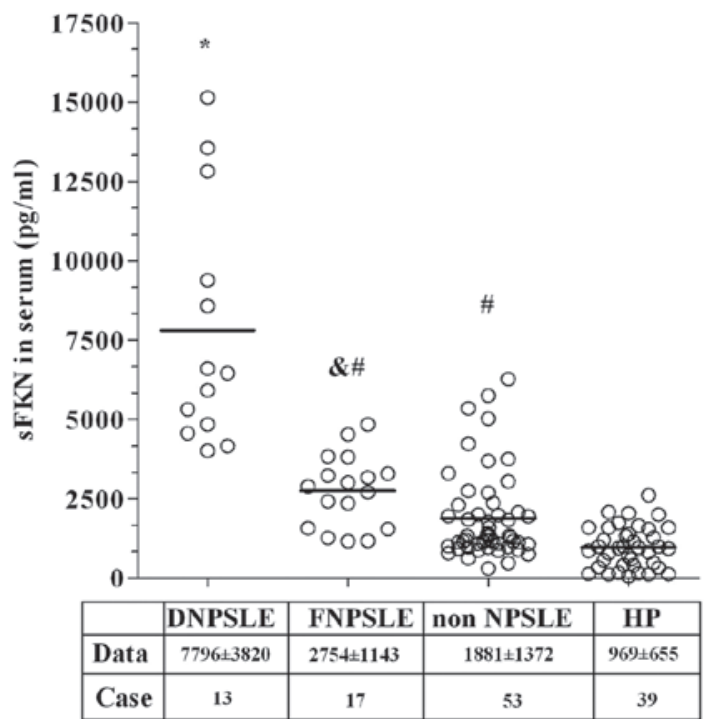

Figure 2. Levels of sFKN in serum. ${ }^{*} \mathrm{P}<0.01$ vs. FNPSLE, non-NPSLE or HP; ${ }^{\&} \mathrm{P}<0.05$ vs. non-NPSLE; ${ }^{~} \mathrm{P}<0.01$ vs. HP. sFKN, soluble fractalkine; DNPSLE, diffuse neuropsychiatric systemic lupus erythematosus; FNPSLE, focal neuropsychiatric systemic lupus erythematosus; non-NPSLE, non-neuropsychiatric systemic lupus erythematosus; HP, healthy person.

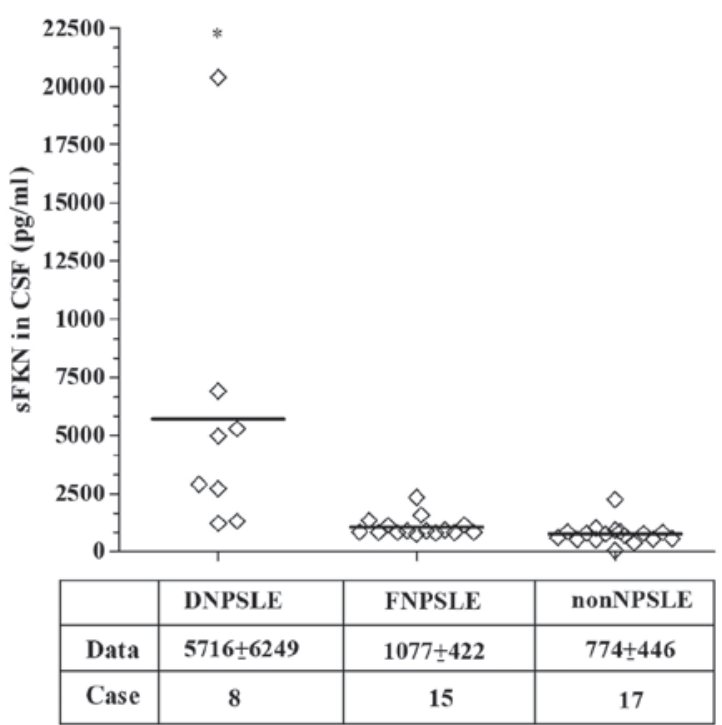

Figure 3. Levels of sFKN in CSF. * $\mathrm{P}<0.01$ vs. FNPSLE and non-NPSLE. sFKN, soluble fractalkine; CSF, cerebrospinal fluid; DNPSLE, diffuse neuropsychiatric systemic lupus erythematosus; FNPSLE, focal neuropsychiatric systemic lupus erythematosus; non-NPSLE, non-neuropsychiatric systemic lupus erythematosus.

the brain tissue of lupus mice were significantly higher than normal mice, while the mRNA expression of FKN was lower than normal mice. By contrast, the former showed a significant difference $(\mathrm{P}=0.028)$ and the latter did not $(\mathrm{P}=0.543$; Fig. 8).

\section{Discussion}

The present study investigated the effect and significance of sFKN and CX3CR1 in NPSLE using the following three parameters: i) mRNA expression of CX3CR1 in PBMCs from human subjects; ii) levels of sFKN in serum and CSF from human subjects; and iii) mRNA expression of FKN 


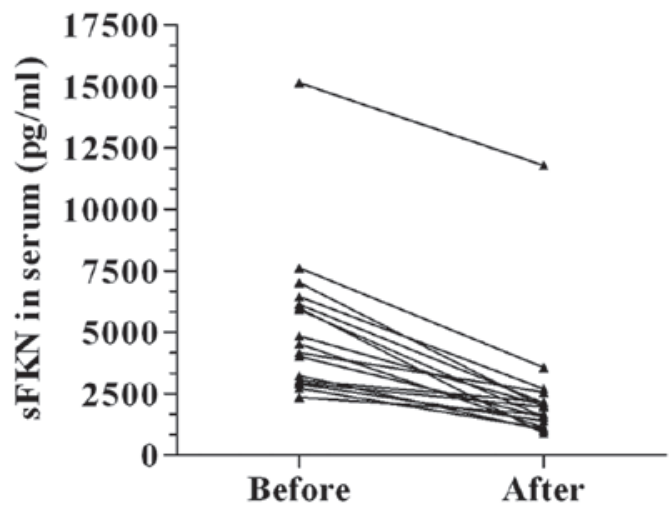

Figure 4. Correlation of sFKN in serum from 17 patients with neuropsychiatric systemic lupus erythematosus prior to and after treatment. sFKN, soluble fractalkine.

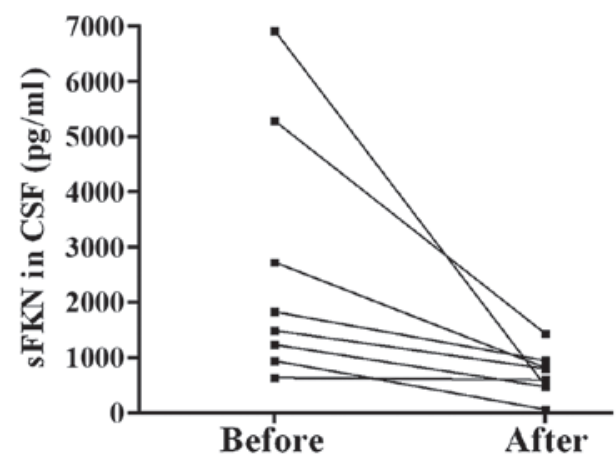

Figure 5. Correlation of sFKN in CSF from 8 patients with neuropsychiatric systemic lupus erythematosus before and after treatment. sFKN, soluble fractalkine; CSF, cerebrospinal fluid.

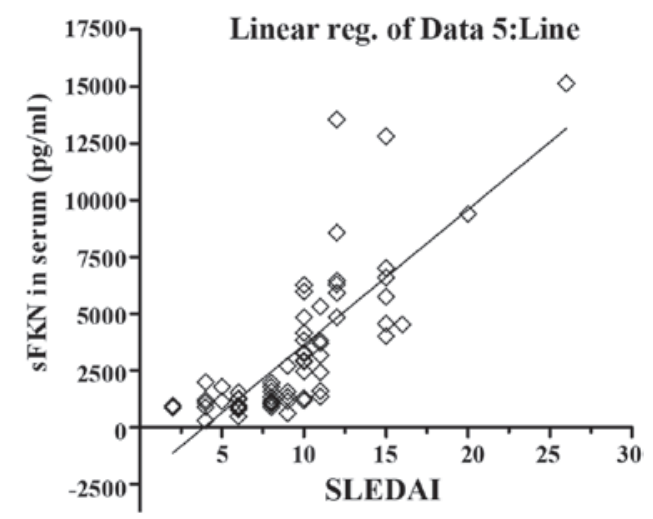

Figure 6. Correlation between sFKN in serum and SLEDAI from patients with SLE. sFKN, soluble fractalkine; SLE, systemic lupus erythematosus; SLEDAI, SLE disease activity index.

and CX3CR1 in the brain tissue of lupus mice. We initially confirmed the significant difference between DNPSLE and FNPSLE patients in their serum levels of sFKN. Furthermore, the mRNA expression of FKN and CX3CR1 in the brain tissue of lupus mice was initially reported in the present study.

The present study found that the mRNA expression of CX3CR1 in PBMCs from patients with SLE and BD was higher than HPs and patients with RA. The pathological basis of SLE and BD is vasculitis, while RA is synovitis (11). The

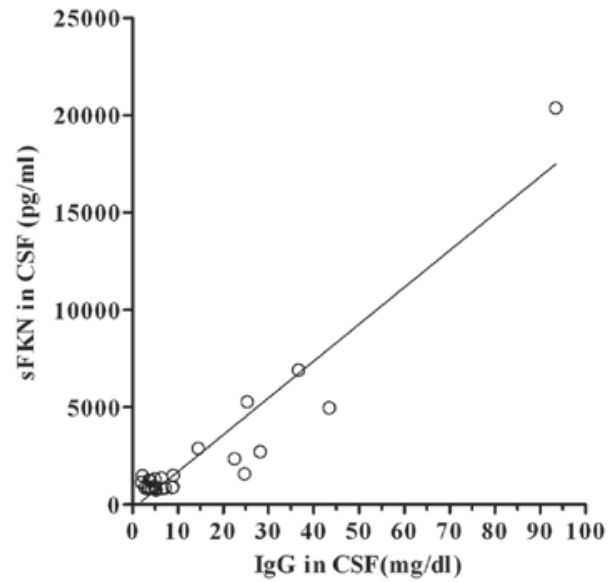

Figure 7. Correlation between $\mathrm{sFKN}$ and $\mathrm{IgG}$ in CSF from patients with neuropsychiatric systemic lupus erythematosus. sFKN, soluble fractalkine; CSF, cerebrospinal fluid.

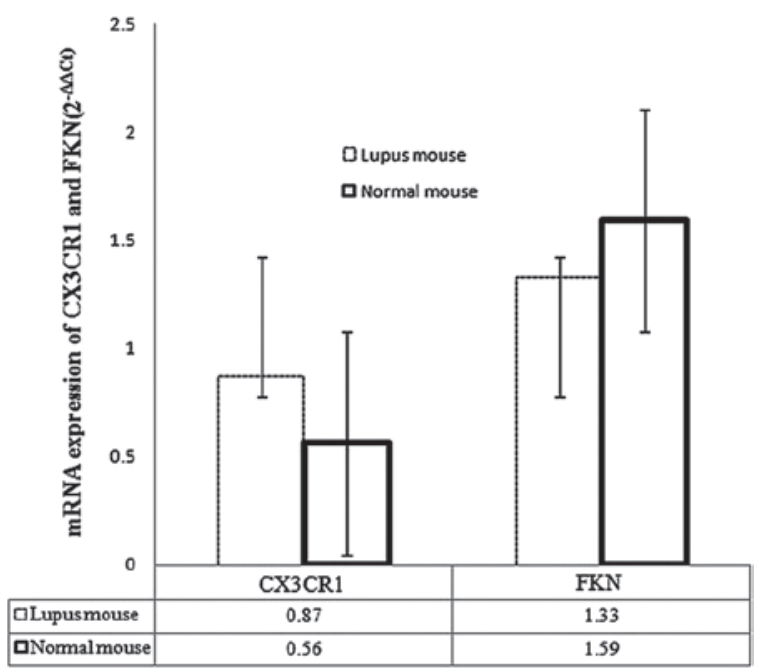

Figure 8. mRNA expression of CX3CR1 and sFKN in brain tissue of lupus mouse and normal mouse. sFKN, soluble fractalkine.

present study found that the mRNA expression of CX3CR1 in PBMCs from patients with SLE and BD was higher than HPs and patients with RA. This result indicated that the expression of CX3CR1, which is the receptor of FKN, increased in vasculitis. Matsunawa et al found that the levels of sFKN in serum from patients with RA combined with rheumatoid vasculitis (RV) was higher than patients with RA only (12). In addition, Yajima et al similarly found that the serum levels of sFKN in patients with SLE were higher than patients with RA (13). Sato et al found levels of sFKN in serum from patients with SLE were higher than those from RA and primary Sjogren's syndrome, whose pathological feature is inflammation of the exocrine glands (14). Bjerkeli et al confirmed that the mRNA expression of CX3CR1 in PBMCs and levels of sFKN in the serum of patients with granulomatosis with polyangiitis (GPA) were both higher than HPs (15). Matsunawa et al discovered serum levels of sFKN in patients with microscopic polyangiitis (MPA) were also higher than HPs (16). Thus, FKN and CX3CR1 may serve a crucial function in SLE, the pathological cause of which vasculitis. Vascular endothelial cells have been 
shown to be activated by interleukin-1 $\beta$, tumor necrosis factor (TNF)- $\alpha$, interferon (IFN)- $\gamma$ and lipopolysaccharide $(17,18)$. Moreover, the nuclear factor- $\kappa \mathrm{B}$ pathway was activated, expression of FKN increased on the surface of vascular endothelial cells, which is known as membrane-anchored FKN $(17,18)$. If membrane-anchored FKN released from the cytomembrane, it was called soluble FKN (sFKN). Membrane-anchored FKN functions as an adhesion molecule and sFKN as a chemotactic factor (19). They captured natural killer cells, monocytes cell and T cells, which can express CX3CR1 on their surfaces (20). In order to drive them near endothelial cells of vessel wall and induce transmembrane transport. These inflammatory cells can release IFN- $\gamma$, TNF- $\alpha$, perforin and granzyme following activation, which can damage vascular endothelial cells and other tissue cells, causing vasculitis and organ damage (21).

The present study found levels of sFKN in serum from patients with SLE were higher than those in serum from HPs, which is consistent with previous reports $(13,14)$. NPSLE was significantly higher than non-NPSLE, which was consistent with a previous report by Sato et al (14), but conflicted with the results of a report by Yajima et al (13). Levels of sFKN in serum and CSF from patients with DNPSLE were significantly higher than those with FNPSLE, particularly patients with clinical manifestations of acute confusion states, cognitive dysfunction and psychosis. A prior study found that sFKN levels in CSF from patients with HIV combined with cognitive dysfunction was higher than patients without cognitive dysfunction (22) and a similar results was observed in the present study except in patients with various types of NPSLE. However, the results of Yajima et al showed sFKN in CSF from patients with FNPSLE was not significantly higher than in patients with DNPSLE (13), which may be due to the small size of the study sample. Moreover, the present study showed that sFKN levels in CSF from patients with DNPSLE was significantly higher than that of non-NPSLE patients, which was different with results obtained by Sato et al (14). Thus, sFKN may serve a crucial function in the pathogenesis of neuropsychiatric symptoms in DNPSLE. In the present study, sFKN levels in serum from patients with SLE were positively correlated with SLEDAI. Furthermore, sFKN levels in CSF from patients with NPSLE were positively correlated with IgG. sFKN in serum and CSF decreased markedly after treatment. These results suggested levels sFKN in the serum and CSF were consistent with disease activity and can be used as an index to evaluate disease activity of SLE and guide its treatment.

Upon the first detect of FKN in the nerve tissues of mice, it was called neurotactin (23). Reports regarding the distribution of FKN and CX3CR1 in the central nervous system of human, mouse and rat differ (23-25). The majority of studies have shown that the expression of FKN is elevated in neurons under physiological conditions, while CX3CR1 is predominantly expressed in microglia, which are a type of mononuclear macrophage in the central nervous system $(26,27)$. Under physiological conditions, FKN can inhibit the activation of microglia and the release of inflammatory factors to protect brain tissue $(28,29)$. Under pathological conditions, active microglia can release excitatory neurotoxins such as glutamic acid to stimulate in part the release of FKN from neurons (30), which was called sFKN. sFKN induced microglia to migrate to inflammatory site, and expression of FasL in microglia and
Fas in brain cell increased simultaneously (31). The Fas/FasL pathway can result in brain cell apoptosis. By contrast, FKN is able to block the Fas/FasL pathway and inhibit apoptosis of microglia by interacting with CX3CR1 on microglia (32). Thus, microglia can continuously release toxic substances such as free radicals, nitric oxide, TNF- $\alpha$ and IL- $1 \beta$ which can aggravate the inflammatory reaction. In the present study, mRNA expression levels of CX3CR1 in brain tissue of lupus mice was significantly higher than in normal mice, suggesting that FKN and CX3CR1 were involved in pathogenesis of NPSLE through local action in the brain tissue. Harrison et al observed that the proliferation of microglia in the nucleus of facial nerve was associated with significantly increasing mRNA expression of CX3CR1 following myotomy of rat facial nerve axons (27). Furthermore, Jiang et al detected increased mRNA expression of CX3CR1 in the lumbar spinal cord from experimental autoimmune encephalomyelitis rats (33). These previous reports have shown that FKN-CX3CR1 were involved in injury of nervous system. As sFKN can function as a chemotactic factor, unlike membrane-anchored FKN, we hypothesized that FKN activity was regulated by modification after translation rather than transcription in the inflammation of nervous system. The mRNA expression levels of FKN in the present study were lower than normal mice, which was consistent with the results of Harrison et al (27), but there was no statistical significance in our study. This result was consistent with the finding that decreased mRNA expression of FKN can weaken protective effect on nerve tissue under pathological conditions.

In the present study, the results showed that FKN and CX3CR1 are crucially involved in the pathogenesis of vasculitis, including SLE and BD. Moreover, the present study indicates that FKN and CX3CR1 may participate in the pathogenesis of NPSLE, particularly DNPSLE. Future studies are required to evaluated the expression of FKN and CX3CR1 in various types of systemic vasculitis, such as Takayasu arteritis, polyarteritis nodosa, BD and anti-neutrophil cytoplasmic antibody associated vasculitis. It has been shown that levels of sFKN in serum from patients with GPA and MPA were increased $(15,16)$. However, Ceyla et al found that $\mathrm{sFKN}$ levels in serum were not changed in active and inactive BD and neuro-BD (34). Therefore, further large-scale studies are required to assess the role of FKN/CX3CR1 in patients with systemic vasculitis. Numerous experiments have shown that antagonism of FKN or CX3CR1 can inhibit inflammation (5,35-38). It is thus possible that FKN-CX3CR1 may be a novel target in the treatment of SLE and systemic vasculitis.

\section{Acknowledgements}

The authors thank Dr Yubo Cai and Dr Hui Zheng for helping in specimen collection. Dr Bing Yan provided lupus mice. Funding was provided by the projects of the construction of key disciplines (grant no. T0203; Shanghai, China).

\section{References}

1. Hochberg MC: Updating the American college of rheumatology revised criteria for the classification of systemic lupus erythematosus. Arthritis Rheum 40: 1725, 1997. 
2. The American College of Rheumatology nomenclature and case definitions for neuropsychiatric lupus syndromes. Arthritis Rheum 42: 599-608, 1999.

3. Foster AM, Baliwag J, Chen CS, Guzman AM, Stoll SW, Gudjonsson JE, Ward NL and Johnston A: IL-36 promotes myeloid cell infiltration, activation and inflammatory activity in skin. J Immunol 192: 6053-6061, 2014.

4. Bazan JF, Bacon KB, Hardiman G, Wang W, Soo K, Rossi D, Greaves DR, Zlotnik A and Schall TJ: A new class of membrane-bound chemokine with a CX3C motif. Nature 385: 640-644, 1997.

5. Nakatani K, Yoshimoto S, Iwano M, Asai O, Samejima K, Sakan H, Terada M, Hasegawa H, Nose M and Saito Y: Fractalkine expression and CD16+ monocyte accumulation in glomerular lesions: association with their severity and diversity in lupus models. Am J Physiol Renal Physiol 299: F207-F216, 2010.

6. Yoshimoto S, Nakatani K, Iwano M, Asai O, Samejima K, Sakan H, Terada M, Harada K, Akai Y, Shiiki H, et al: Elevated levels of fractalkine expression and accumulation of CD16+ monocyte in glomerui of active lupus nephritis. Am J Kidney Dis 50: 47-58, 2007.

7. Arnett FC, Edworthy SM, Bloch DA, McShane DJ, Fries JF, Cooper NS, Healey LA, Kaplan SR, Liang MH, Luthra HS, et al: The American rheumatism association 1987 revised criteria for the classification of rheumatoid arthritis. Arthritis Rheum 31: 315-324, 1988.

8. Criteria for diagnosis of Behçet's disease. International study group for Behçet's disease. Lancet 335: 1078-1080, 1990.

9. Zhang J, Wu J, Zhao F, Yan Yand Zhao Z: Effect of different temperature and different stored time on concentration and purity of total RNA isolation. Shi Yan Ji Shu Yu Guan Li 11: 79-82, 2013 (In Chinese).

10. Formiga F, Moga I, Pac M, Mitjavila F, Rivera A and Pujol R: Mild presentation of systemic lupus erythematosus in elderly patients assessed by SLEDAI. Lupus 8: 462-465, 1999.

11. Boissier MC, Semerano L, Challal S, Saidenberg-Kermanac'h N and Falgarone G: Rheumatoid arthritis: from autoimmunity to synovitis and joint destruction. J Autoimmunity 39: 222-228, 2012.

12. Matsunawa M, Isozaki T, Odai T, Yajima N, Takeuchi HT, Negishi M, Ide H, Adachi M and Kasama T: Increased serum levels of soluble fractalkine (CX3CL1) correlate with disease activity in rheumatoid vasculitis. Arthritis Rheum 54: 3408-3416, 2006.

13. Yajima N, Kasama T, Isozaki T, Odai T, Matsunawa M, Negishi M, Ide H, Kameoka Y, Hirohata S and Adachi M: Elevated levels of soluble fractalkine in active systemic lupus erythematosus: Potential involvement in neuropsychiatric manifestations. Arthritis Rheum 52: 1670-1675, 2005.

14. Sato E, Iikuni N, Yoshio T, Minota S, Kamatani N and Okamoto H: Soluble fractalkine in the cerebrospinal fluid of patients with neuropsychiatric lupus. Ann Rheum Dis 65: 1257-1259, 2006.

15. Bjerkeli V, Damås JK, Fevang B, Holter JC, Aukrust P and Frøland SS: Increased expression of fractalkine (CX3CL1) and its receptor, CX3CR1, in Wegener's granulomatosis-possible role in vascular inflammation. Rheumatology (Oxford) 46: 1422-1427, 2007.

16. Matsunawa M, Odai T, Wakabayashi K, Isozaki T, Yajima N, Miwa Y, Nohtomi K, Takeuchi H and Kasama T: Elevated serum levels of soluble CX3CL1 in patients with microscopic polyangiitis. Clin Exp Rheumatol 27: 72-78, 2009.

17. Sung MJ, Kim DH, Davaatseren M, Hur HJ, Kim W, Jung YJ, Park SK and Kwon DY: Genistein suppression of TNF-alpha-induced fractalkine expression in endothelial cells. Cell Physiol Biochem 26: 431-440, 2010.

18. Garcia GE, Xia Y, Chen S, Wang Y, Ye RD, Harrison JK, Bacon KB, Zerwes HG and Feng L: NF-kappaB-dependent fractalkine induction in rat aortic endothelial cells stimulated by IL-1beta, TNF-alpha and LPS. J Leukoc Biol 67: 577-584, 2000.

19. Patel S, Mukovozov I and Robinson LA: Assessment of the recycling of the membrane-bound chemokine, $\mathrm{CX}_{3} \mathrm{CL} 1$. Methods Mol Biol 748: 143-153, 2011.
20. Fong AM, Robinson LA, Steeber DA, Tedder TF, Yoshie O, Imai $\mathrm{T}$ and Patel DD: Fractalkine and CX3CR1 mediate a novel mechanism of leukocyte capture, firm adhesion, and activation under physiologic flow. J Exp Med 188: 1413-1419, 1998.

21. Goda S, Imai T, Yoshie O, Yoneda O, Inoue H, Nagano Y, Okazaki T, Imai H, Bloom ET, Domae $\mathrm{N}$ and Umehara $\mathrm{H}$ : CX3C-chemokine, fractalkine-enhanced adhesion of THP-1 cells to endothelial cells through integrin-dependent and -independent mechanisms. J Immunol 164: 4313-4320, 2000.

22. Erichsen D, Lopez AL, Peng H, Niemann D, Williams C, Bauer M, Morgello S, Cotter RL, Ryan LA, Ghorpade A, et al: Neuronal injury regulates fractalkine: Relevance for HIV-1 associated dementia. J Neuroimmunol 138: 144-155, 2003.

23. Pan Y, Lloyd C, Zhou H, Dolich S, Deeds J, Gonzalo JA, Vath J, Gosselin M, Ma J, Dussault B, et al: Neurotactin, a membrane-anchored chemokine upregulated in brain inflammation. Nature 387: 611-617, 1997.

24. Meucci O, Fatatis A, Simen AA and Miller RJ: Expression of CX3CR1 chemokine receptors on neurons and their role in neuronal survival. Proc Natl Acad Sci USA 97: 8075-8080, 2000.

25. Maciejewski-Lenoir D, Chen S, Feng L, Maki R and Bacon KB: Characterization of fractalkine in rat brain cells: migratory and activation signals for CX3CR-1-expressing microglia. J Immunol 163: 1628-1635, 1999.

26. LeeS, Varvel NH, Konerth ME,Xu G, Cardona AE, RansohoffRM and Lamb BT: CX3CR1 deficiency alters microglial activation and reduces beta-amyloid deposition in two Alzheimer's disease mouse models. Am J Pathol 177: 2549-2562, 2010.

27. Harrison JK, Jiang Y, Chen S, Xia Y, Maciejewski D, McNamara RK, Streit WJ, Salafranca MN, Adhikari S, Thompson DA, et al: Role for neuronally derived fractalkine in mediating interactions between neurons and CX3CR1-expressing microglia. Proc Natl Acad Sci USA 95: 10896-10901, 1998.

28. Lyons A, Lynch AM, Downer EJ, Hanley R, O'Sullivan JB, Smith A and Lynch MA: Fractalkine-induced activation of the phosphatidylinositol-3 kinase pathway attentuates microglial activation in vivo and in vitro. J Neurochem 110: 1547-1556, 2009.

29. Mizuno T, Kawanokuchi J, Numata K and Suzumura A: Production and neuroprotective functions of fractalkine in the central nervous system. Brain Res 979: 65-70, 2003.

30. Chapman GA, Moores K, Harrison D, Campbell CA, Stewart BR and Strijbos PJ: Fractalkine cleavage from neuronal membranes represents an acute event in the inflammatory response to excitotoxic brain damage. J Neurosci 20: RC87, 2000.

31. Vogt M, Bauer MK, Ferrari D and Schulze-Osthoff K: Oxidative stress and hypoxia/reoxygenation trigger CD95 (APO-1/Fas) ligand expression in microglial cells. FEBS Lett 429: 67-72, 1998.

32. Boehme SA, Lio FM, Maciejewski-Lenoir D, Bacon KB and Conlon PJ: The chemokine fractalkine inhibits Fas-mediated cell death of brain microglia. J Immunol 165: 397-403, 2000.

33. Jiang Y, Salafranca MN, Adhikari S, Xia Y, Feng L, Sonntag MK, deFiebre CM, Pennell NA, Streit WJ and Harrison JK: Chemokine receptor expression in cultured glia and rat experimental allergic encephalomyelitis. J Neuroimmunol 86: 1-12, 1998.

34. Ceyla I, Tuba T, Esra A, Banu O, Isil F, Ali GM, Turgut I and Banu B: Fractalkine (CX3CL1) levels in patients with Behçet's disease and neuro-Behçet's disease. J Neurol Sci 315: 120-122, 2012.

35. Mattison HA, Nie H, Gao H, Zhou H, Hong JS and Zhang J: Suppressed pro-inflammatory response of microglia in CX3CR1 knockout mice. J Neuroimmunol 257: 110-115, 2012.

36. Feng L, Chen S, Garcia GE, Xia Y, Siani MA, Botti P, Wilson CB, Harrison JK and Bacon KB: Prevention of crescenticglomerolonephritis by immunoneutralization of the fractalkine receptor CX3CR1 rapid communication. Kidney Int 2: 612-620, 1999.

37. Inoue A, Hasegawa $\mathrm{H}$, Kohno M, Ito MR, Terada M, Imai $\mathrm{T}$, Yoshie $\mathrm{O}$, Nose $\mathrm{M}$ and Fujita $\mathrm{S}$ : Antagonist of fractalkine(CX3CL1) delays the initiation and ameliorates the progression of lupus nephritis in MRL/lpr mice. Arthritis Rheum 52: 1522-1533, 2005.

38. Dorgham K, Ghadiri A, Hermand P, Rodero M, Poupel L, Iga M, Hartley $\mathrm{O}$, Gorochov $\mathrm{G}$, Combadière $\mathrm{C}$ and Deterre P: An engineered CX3CR1 antagonist endowed with anti-inflammatory activity. J Leukoc Biol 86: 903-911, 2009. 